\title{
Los sistemas de control interno en las Mipymes y su impacto en la efectividad empresarial
}

\author{
Internal control systems at SME's and their impact in business effectiveness
}

Recibido: 23/06/14 - Aprobado versión final: 11/09/14

\section{Luz Idalia Castañeda Parra*}

Resumen: el presente artículo es el resultado de una investigación de tipo analítico y descriptivo, en la que se llevó a cabo un estudio comparativo con tres empresas del sector confección de la ciudad de Medellín, una mediana, una pequeña y una micro, con el fin de precisar la contribución del sistema de control interno al logro de los objetivos de estas organizaciones y presentar los lineamientos de un sistema de control interno organizacional adecuado a las Mipymes en cuestión, utilizando como referencia el Modelo COSO.

Palabras clave: Mipymes, efectividad empresarial, control interno, Modelo COSO.

Abstract: this paper is the outcome of an analysis- and description-based research. It
carried out a comparative study with three clothing businesses in the city of Medellín
- one medium, another small and one more micro. This intended to establish the
contribution of the internal control system to the achievement of these organizations'
goals, and to show the guidelines for an organizational internal control system that is
appropriate for the above mentioned SME's, by using COSO model as a reference.

Keywords: SME's, enterprise effectiveness, internal control, COSO model.

JEL: M42

* Contadora Pública y Magíster en Ciencias Contables de la Universidad de Antioquia, Medellín - Colombia. lcastanedaudea@gmail.com 


\section{Conterto castañedat L}

\section{Les systèmes de contrôle interne dans les PME du secteur de la confection de la ville de Medellin et leur effet sur la performance de ces dernières}

Résumé: dans cette recherche nous entreprenons une étude sur la structure des entreprises du secteur de la confection de Medellin. Cela afin de préciser la contribution du système de contrôle interne à l'obtention des objectifs des entreprises relatifs à leur organisation. En même temps nous envisageons de présenter les fondements d'un système de contrôle interne approprié aux PME en question basé sur le Modèle COSO.

Mots clé: PME, performance des entreprises, contrôle interne, Modèle COSO.

\section{Introducción}

Actualmente, el entorno empresarial está marcado por la incertidumbre y el riesgo a causa de la competencia, la internacionalización de los mercados, la disminución de las barreras comerciales, el acceso a la información y los avances tecnológicos. Lo anterior ha provocado cambios importantes en la forma como las empresas gestionan. En estas condiciones, la gestión de las empresas se ha convertido en una cuestión fundamental para su supervivencia, ya que las necesidades empresariales más inmediatas son adaptarse a los cambios permanentes a fin de lograr cada vez un mejor posicionamiento en los mercados y aprovechar las oportunidades para el logro del éxito.

El problema para la dirección empresarial hoy no es simplemente entender la necesidad de cambio sino cómo efectuarlo de forma tal que se logren los objetivos a través de las estrategias diseñadas. En este sentido, el control de gestión en las empresas debe conducir a que los componentes operativos les permitan alcanzar sus objetivos estratégicos. En especial para las empresas micro, pequeñas y medianas, denominadas Mipymes, las cuales para sobrevivir en este contexto se han visto obligadas a formalizar sus procesos de planificación y de control de todas sus operaciones.

En relación con lo anterior, este artículo de investigación expone un estudio comparativo con tres empresas del sector confección de la ciudad de Medellín, una mediana, una pequeña y una micro, con el fin de precisar la contribución del sistema de control interno al logro de los objetivos estratégicos y operativos en la organización, tomando como referencia el marco conceptual de control interno más reconocido, propuesto en 1992 por el Committe of Sponsoring Organizations of the Treadway Commission, conocido generalmente como "Modelo COSO". 
Es importante anotar que en el año 2002 se publicó el Modelo COSO Enterprise Risk Management o ERM -, que no trata sobre control interno sino que lo complementa, está basado en la gestión de riesgos de la administración y hace parte de una investigación que adelanta la autora sobre la administración de riesgos en las pymes del clúster textil/confección en Antioquia.

Por tanto, el principal propósito de este artículo de investigación es, precisamente, determinar la efectividad del sistema de control interno en el cumplimiento de los objetivos organizacionales de las mipymes objeto de estudio, de tal manera que a partir de los resultados de la evaluación del control interno en estas mipymes se puedan presentar algunas recomendaciones al modelo de control interno: que se adapte a sus necesidades, que garantice una seguridad razonable frente a la protección de sus activos, que genere eficiencia y eficacia en sus operaciones de confiabilidad a la información financiera y, por supuesto, que dé cumplimiento a leyes y normas aplicables.

En este orden de ideas, se entenderá que el cumplimiento de los objetivos de control interno favorece la supervivencia y el éxito de las organizaciones, al ser directamente vinculados con su razón de ser, y por tanto se espera que los resultados evidenciados sirvan de referente para futuras investigaciones, y también a las mipymes que vayan a someterse al proceso de formalización de sus sistemas de control interno, para lo cual es fundamental difundir las investigaciones y trabajos de campo, y concientizar a estas empresas sobre la importancia de los aspectos presentados en este estudio.

\section{Metodología}

El presente artículo compone una investigación de tipo analítico y descriptivo. Analítico porque busca descomponer el objeto de estudio en cada una de sus partes para estudiarlas en forma individual (Bernal, 2006, p. 112), y descriptivo porque se reseñan las características de la situación objeto de estudio (Salkind, 1998, p. 12). En ella se busca conocer las características que identifican los elementos y componentes del control interno en las mipymes y la percepción que los administradores tienen del cumplimiento de los objetivos organizacionales, en función de la aplicación o no del control interno. La metodología aplicada a este proceso de investigación, el cual abarca componentes que en este artículo no se precisan debido a su nivel de profundidad y alcance, se estructura a partir de un tipo de investigación mixta que se expresa en la Figura 1. 


\section{Contexto castañeda, L.}

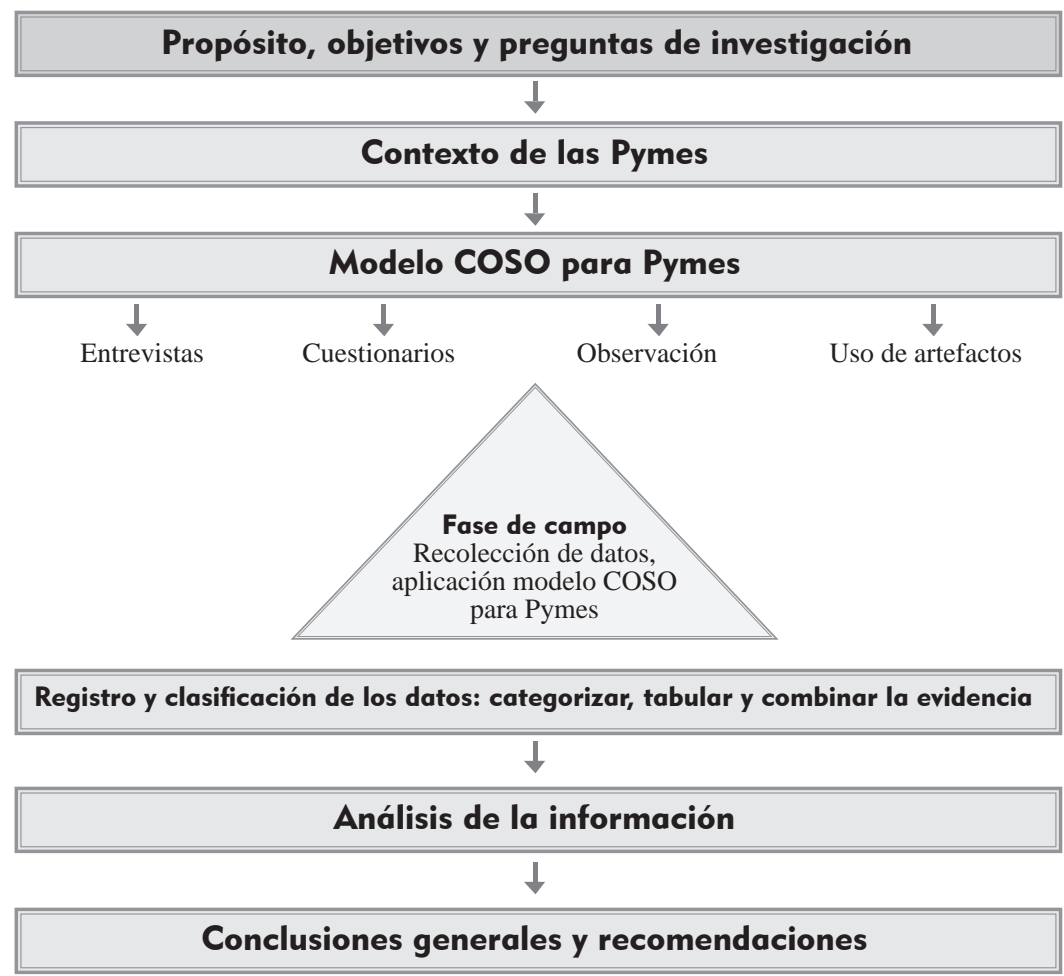

Figura 1. Diseño metodológico

Fuente: elaboración a partir de Villareal \& Landeta (2010).

\section{Resultados}

\section{Problemática de las pymes en Colombia}

Como se desprende de la sección anterior, las pymes tienen debilidades estructurales en cuanto a la falta de herramientas para crear estrategias que permitan una gestión para proyectar la empresa en el mediano y largo plazo, les falta planeación, lo que se convierte en una limitante para la inserción en el ámbito internacional poniendo en riesgo su continuidad en el mercado nacional. Así como el difícil acceso a líneas de crédito, por lo cual no es posible la inversión en tecnología, en capital de trabajo y en "know how", su gestión administrativa, financiera, contable y operativa es muy informal y se hace de manera intuitiva y no utilizan la contabilidad para la toma de decisiones. (Beltrán, Torres, Camargo, \& Bello, 2004, p. 180). 
Otra falencia es que adolecen de la gestión necesaria para convertirse en unidades innovadoras, autosostenibles y en crecimiento (Vargas, 2003, p. 98). En la parte gerencial tienen desconocimiento administrativo en cuanto al manejo del recurso humano y a los procesos operativos. Gran parte de estas empresas se han creado por la experiencia de empleados que trabajaron en otras empresas, y aportan al sector su experiencia y conocimiento técnico, pero no el administrativo. Aunado a esto, está la desinformación sobre las oportunidades para exportar de forma competitiva y sostenible, como el resultado de una capacitación básica y de una gestión gerencial para incursionar en el comercio exterior. (Andriani, Biasca \& Rodríguez, 2004)

En un estudio se muestra que la baja densidad empresarial en Antioquia se explica a su vez por la alta mortalidad empresarial que se registra en la región, pues de 100 empresas constituidas, desaparecen 29 entre el primero y el tercer año de constitución; 42 en el séptimo año, y al décimo año han desaparecido un total de 47. (Cámara de Comercio de Medellín, 2009)

Este estudio también menciona que la informalidad de las empresas no es solamente por el incumplimiento de lo ordenado por la ley sino por una estructura empresarial frágil y vulnerable ante los sucesivos cambios del entorno que traen consigo nuevas exigencias para los mercados a las que no están en capacidad de responder. No tienen un direccionamiento claro, no saben lo que esperan sus clientes, trabajan de manera reactiva resolviendo los problemas conforme surgen, hacen nuevas inversiones en líneas de productos o departamentos sin la adecuada planeación, y además tienen desorden en los procesos y en las tareas de cada área, lo que hace que éstas sean redundantes y repetitivas.

La Fundación Corona evidenció la reducida dimensión de las microempresas, en aspectos permanentes como: la excesiva dependencia de sus fondos propios, la restricción al acceso del crédito bancario, los reducidos mercados, el poco desarrollo tecnológico, la baja calificación de la fuerza de trabajo, limitaciones para aplicar nuevas técnicas de gestión, precario diseño organizativo y limitaciones para exportar. (Fundación Corona, 2005)

Para Puyana y Matallana (2002, p.67) una pyme se crea con un mínimo de recursos y el mayor o menor conocimiento respecto a una actividad o un mercado, que en algunas ocasiones resultan "suficientes" para el emprendimiento, pero no para que la empresa se mantenga y se convierta en una empresa innovadora y competitiva. La dificultad para conseguir fuentes de financiamiento se deriva regularmente de la poca claridad que tienen sobre los objetivos y las metas que se desean alcanzar, y de la ausencia de planes estratégicos que generen un escenario ordenado y acorde con el plan de negocio. 


\section{Contexto castaredta L}

En este sentido, es evidente la falta de modelos de gestión en lo financiero y organizacional que se adapten a las condiciones particulares de las pequeñas empresas. Para implementarlos se requiere trascender los paradigmas de gestión "artesanales", de modo que estas unidades económicas se administren con una visión de empresa y se logre su consolidación individual, sectorial y regional. (Correa \& Jaramillo, 2007, p.93)

Los anteriores planteamientos permiten visualizar debilidades de las pymes, tanto jurídicas como administrativas y financieras, que son el punto de partida para esclarecer una situación que se presenta desde hace mucho tiempo pero que sólo en los últimos años se ha convertido en tema de investigación y discusión, especialmente en el ámbito empresarial.

A partir de estas debilidades es que la gerencia de una empresa debe buscar la construcción de nuevos modelos que permitan lograr los objetivos organizacionales, ser competitivas, productivas, innovadoras, sostenibles y con responsabilidad social.

La noción que aquí se plantea es un paso adelante para las empresas en general, ya que con ello se podría hacer uso de nuevos modelos de control con los cuales tomar decisiones que cubran sus necesidades y ayuden a mejorar el interior de la organización junto con el entorno que la rodea. Desde esta perspectiva, las pymes colombianas deben alinearse con las tendencias que impone la gerencia del siglo XXI, en la que competitividad se convierte en el eje central del negocio y la generación de conocimiento en el factor determinante para transformar este tipo de empresas y promover la creación de productos y servicios con valor agregado y pertinencia social.

Las pymes deben reinventar su modelo de negocio basadas en una fuerza productiva que contemple una estructura corporativa y competitiva centrada en la cultura de la planificación, el talento humano calificado, la flexibilidad y adaptabilidad del negocio, el marketing, las tecnologías de punta, la asociatividad empresarial, y la estimulación del pensamiento estratégico de sus gerentes para que puedan sostener sus ventajas empresariales girando en torno a la frontera de la competitividad. (Villegas \& Toro, 2010, p. 86)

Hoy en día es evidente la urgencia de plantear alternativas y soluciones que estructuren un sistema para comprender la realidad de las pequeñas empresas, en donde se evalúen de manera permanente los riesgos que se presentan en su entorno y ambiente organizacionales, y que impiden su desarrollo, competitividad, productividad y sostenibilidad. Esto con el fin de fortalecer en ellas estas actividades por medio de estrategias adecuadas que tengan en cuenta la innovación tecnológica y el cambio como elementos fundamentales para su desarrollo. 
Las organizaciones deben establecer un mínimo de reglas de operatividad para lograr sus objetivos, que se denominan sistema de control interno, el cual está sujeto a regulaciones nacionales e internacionales, pero cuya puesta en marcha y óptimo funcionamiento son responsabilidad de la administración de cada organización.

Tomando en cuenta los anteriores argumentos, es deducible que los modelos de control interno no responden a las necesidades organizacionales o, al parecer, no se están teniendo en cuenta en toda su dimensión, ya que las empresas se han constituido, más por un hacer causado por una necesidad económica basadas sólo en la experiencia adquirida con anterioridad, que por estudios minuciosos de planes de negocios y de diseño organizacional, lo que puede incidir en su eficiencia y eficacia, y además pone en riesgo la posibilidad de permanecer en el mercado (Viloria, 2005, p. 87). El tamaño de estas empresas facilita su adaptación al cambio en lo que tiene que ver con su capacidad gerencial, formalización, gobierno corporativo, acceso a nuevas tecnologías, utilización de financiamiento, capacitación de recurso humano, cultura de emprendimiento, innovación y diseño de sistemas de seguimiento y evaluación. (Ferraro, 2011)

\section{El Control Interno basado en el Modelo COSO}

$\mathrm{Al}$ retomar el informe del Committee of Sponsoring Organizations - COSO (1997), este enfatiza que el sistema de control interno es una herramienta de la administración pero no un sustituto para ésta, y que los controles se deberán ejecutar dentro de las actividades de operación y no fuera de ellas.

La primera categoría se orienta a los objetivos básicos de negocios de una entidad, que incluyen los objetivos de desempeño, rentabilidad y salvaguarda de los recursos. La segunda se relaciona con la preparación y publicación de estados financieros confiables. La tercera se refiere al cumplimiento de aquellas leyes y regulaciones a las que está sujeta la empresa. El logro de los objetivos de control interno aporta a la supervivencia y al éxito de las organizaciones. Siguiendo a Dorta (2005, p.9), el nuevo concepto introducido por COSO rompe con las acepciones tradicionales, al asumir que el control interno es una práctica social desarrollada en todos los ámbitos organizacionales; esto es, un proceso o una multiplicidad de procesos omnipresentes en la planificación, la dirección y la gestión de la entidad e inherentes a ella.

La consideración del riesgo como uno de los componentes fundamentales del control interno es ampliada en la versión del informe COSO 2004, en el cual se definen cuatro categorías de objetivos, es decir que antepone el objetivo "estrategia" a los tres ya conocidos: operacionales, de información y de cumplimiento normativo. En síntesis, "la estrategia busca el logro de las metas de alto nivel, las cuales están alineadas para apoyar la misión y 


\section{Contexto Castañeda, L.}

la visión de empresa" (Canetti, 2005, p. 74). El control interno se define como un proceso que es llevado a cabo por personas de diferentes niveles jerárquicos que deben aportar compromiso e involucrarse en el proceso, "que aportará solamente un grado razonable de seguridad y jamás se debe esperar la seguridad total" (Hourcle, 2000). En este sentido, el control interno constituye un sistema interactuante, un proceso que involucra a todo el recurso humano de la organización más allá de los manuales de normas y procedimientos, bajo la responsabilidad de la gerencia de la entidad.

El control interno consta de cinco componentes interrelacionados, integrados al proceso administrativo, derivados de la manera como la administración realiza los negocios. Estos componentes se aplican a las grandes empresas, pero es importante que las pequeñas y medianas los implementen de acuerdo con sus propias exigencias, pues sus controles pueden ser menos formales y estructurados, pero efectivos. Estos componentes son: ambiente de control, evaluación de riesgos, actividades de control, información, y comunicación y monitoreo. (COSO, 1997)

\section{Ambiente de control}

El informe COSO (1997) señala que el entorno de control marca las pautas de comportamiento de una organización y constituye la base de todos los demás componentes, pues es el elemento que proporciona disciplina y estructura. El ambiente de control se determina de acuerdo con la integridad y competencia del personal de una organización; igualmente, los valores éticos son un elemento esencial que afecta otros componentes del control. Entre sus factores se incluye la filosofía de la administración, la atención y la guía proporcionadas por el consejo de administración, y el estilo operativo, así como la manera en que la gerencia confiere autoridad, asigna responsabilidades y organiza a su personal. Desde una perspectiva empírica, los diversos elementos del entorno de control constituyen los factores más importantes para lograr un control efectivo. (Cohen, 2000, citado por Dorta, 2005)

El modelo estadounidense reconoce la necesidad de ampliar el modelo estructural funcionalista de los sistemas de control, para lo cual incorpora la idea de la cultura organizativa, y se refiere a ella como los elementos que permiten alcanzar la integridad de la organización y facilitan la congruencia entre el comportamiento individual y organizativo en aras de lograr los objetivos. (Dorta, 2005)

\section{Evaluación de riesgos}

Es la identificación y el análisis de los riesgos que se relacionan con el logro de los objetivos, pues la administración debe cuantificar su magnitud y proyectar la probabilidad de éxito y sus posibles consecuencias si 
no se consiguen. En la dinámica actual de los negocios se debe prestar especial atención a los avances tecnológicos, los cambios en los ambientes operativos, las nuevas líneas de negocios, la reestructuración corporativa, la expansión o adquisiciones extranjeras, el ingreso de personal nuevo y el rápido crecimiento.

El enfoque no se determina en el uso de una metodología particular de evaluación de riesgos sino en la realización de la evaluación de riesgos como una parte natural del proceso de planeación. COSO propone identificar y evaluar los riesgos corporativos tradicionalmente centrados en algunos riesgos financieros como el fraude, el incumplimiento de la normativa financiera o la divulgación de información fidedigna o privada.

Este informe sugiere que el control interno se centre exclusivamente en las actividades de diagnóstico, sin perjuicio de que las mismas queden integradas y acopladas en el proceso de formulación de objetivos y en el desarrollo de acciones correctivas. El control interno es parte del proceso de gestión pero no todas las actividades de la dirección constituyen elementos del mismo.

\section{Actividades de control}

Se definen como "las normas y procedimientos que constituyen las acciones necesarias para implementar las políticas que pretenden asegurar el cumplimiento de las directrices que la dirección ha establecido con el fin de controlar los riesgos".

Las actividades de control ocurren en todos los niveles y en todas las funciones de la organización, lo que incluye los procesos de aprobación y autorización, las iniciativas técnicas, los programas y conciliaciones, entre otros. Las actividades de control se clasifican en controles preventivos, detectivos y correctivos, controles en los manuales de usuario, controles de cómputo o de tecnología de información y controles administrativos.

Las actividades de control deben estar acordes con el tipo de empresa, y además el personal debe realizar cada día una o varias actividades específicas para asegurarse de que la organización se adhiera a los planes de acción y al seguimiento de la consecución de objetivos.

El modelo COSO pretende que las actividades de control se fundamenten en el grado de riesgo y no sobre la base de los posibles errores que las personas puedan cometer, de tal forma que exista una correlación entre la intensidad del riesgo y la actividad de control.

\section{Información y comunicación}

Engloba el conjunto de procedimientos que, cuando se ejecutan, proporcionan información para la toma de decisiones con miras a un buen control de la 


\section{Contexto castañed, L.}

organización. De esta forma se resalta el valor de la información más allá de su tradicional aspecto operativo, y se asume una orientación estratégica, a la que se integra la planificación, el diseño y la implantación de los sistemas de información. También se debe generar información relevante y comunicarla oportunamente, de tal manera que sea entendida para cumplir con sus responsabilidades.

El informe COSO hace énfasis en que la información adquiere su razón de ser en la medida en que permita a las personas cumplir con sus responsabilidades respecto a las tres categorías de objetivos, por lo que un sistema de control interno eficaz debe posibilitar información de calidad y sistemas adecuados de comunicación interna y externa. (Davis \& Militello, 1994)

\section{Monitoreo}

Los controles internos deben ser monitoreados constantemente para asegurar que el proceso se encuentra operando como se planeó y comprobar que son flexibles a los cambios de las situaciones que les dieron origen. El alcance y la frecuencia del monitoreo dependen de los riesgos que se pretenden detectar. Las actividades de monitoreo constante pueden estar inmersas en los procesos del negocio o a través de evaluaciones separadas de la operación, es decir, mediante auditoría interna o externa.

Según el informe COSO, dicho seguimiento puede tomar la forma de actividades de supervisión mediante evaluaciones continuas, periódicas o una combinación de ambas. La supervisión continua "se inscribe en el marco de las actividades corrientes y comprende unos controles regulares realizados por la dirección, así como determinadas tareas que realiza el personal en el cumplimiento de sus funciones". (Dorta, 2005)

Como elementos esenciales de este componente, COSO sugiere que la organización defina quién participa en el proceso de evaluación, cuáles van a ser el proceso y la metodología de la evaluación, así como la forma de comunicar las evidencias detectadas.

\section{Efectividad del control interno}

Para COSO (1997), el control interno se concibe como un proceso interactivo multidireccional en el que todos sus componentes influyen entre sí, dando como resultado un sistema integrado que responde de manera dinámica a las circunstancias cambiantes de los entornos interno y externo de la organización y a los efectos de lograr los objetivos operacionales de información y cumplimiento.

Esta visión sistémica del control interno aporta numerosas posibilidades a la hora de evaluarlo, como que el control interno es fundamental para todas 
las actividades y unidades de la organización o para la empresa en general, y así segmentar la evaluación por unidades departamentales, por áreas o por actividades funcionales. Esta evaluación puede concretarse con el estudio de la idoneidad de uno o varios elementos que integran un componente para observar cómo afectan los objetivos del control interno. Igualmente, se puede analizar en qué medida favorecen o no cada uno de los componentes el logro de alguna de las categorías de objetivos. (Dorta, 2005)

Según el informe COSO (1997), el control interno puede considerarse efectivo en cada una de las tres categorías de objetivos, si el consejo directivo y la administración tienen la certeza de entender que el tiempo en el cual se están consiguiendo los objetivos está acorde con el tipo de operaciones de la entidad, así como con la elaboración y publicación de los estados financieros de forma confiable. Esto para observar si se cumple con las leyes y las regulaciones aplicables.

La efectividad del control interno se mide con el transcurso del tiempo y se determina con un juicio que resulta de evaluar si los cinco componentes se dan y funcionan con una alta seguridad, lo cual es el resultado de la consecución de una o varias de las categorías establecidas. También se entiende como el resultado o el logro obtenido por el cumplimiento de alguno de los objetos de acuerdo con lo planeado, desde un marco flexible $\mathrm{y}$ en un entorno de incertidumbre e imprevisibilidad.

Los controles efectivos son aquellos que "están integrados con el sistema de planificación, son flexibles, precisos, oportunos y objetivos". Con un control efectivo "la organización no tendrá problemas de control a causa de un enfoque incorrecto o [por] ineficiencia" (Palma, 1993). Asimismo, permite hacer un control de la relación costo-beneficio favorable; vale aclarar que el valor del objeto controlado no es superado por el valor de los esfuerzos involucrados en la labor de control.

Según Palma (1993), un control interno efectivo tiene las siguientes características:

Integración con la planeación, o sea que se puedan controlar los aspectos determinados en el plan de la organización.

Flexibilidad en función de los cambios, lo cual deriva en sistemas adaptativos e inteligentes desde las ingenierías, con lo cual se logra cada vez más precisión y oportunidad, pues el control basado en sistemas de información eficientes contribuye a reducir el desconocimiento de las perturbaciones del sistema original o del ente objeto de control.

Es objetivo, es decir, libre de juicios de valor para la interpretación orientada a representar la realidad. 


\section{Conterkto Castañeda, L.}

\section{Análisis de los datos}

Para el análisis de los datos se utilizó la estadística descriptiva. Los resultados se tabularon y graficaron para una mejor comprensión. La información recopilada con el cuestionario se tabuló en excel, con el fin de realizar un análisis basado en promedios aritméticos. Los resultados de la evaluación se registraron en la base de datos que las empresas presentaron en forma de tablas para facilitar su comprensión, donde se calculó el promedio en cada uno de los elementos de cada componente, con base en las respuestas de los dos entrevistados de cada empresa (véase la Tabla 1).

Tabla 1. Escala de valoración de puntos para la evaluación del nivel de efectividad de control interno

\begin{tabular}{ccc}
\hline Media & Control Interno Actual & Nivel de Efectividad \\
\hline Entre 0.0 y 2.0 Puntos & Inexistente & Inefectivo \\
Entre 2.1 y 3.0 Puntos & Deficiente & Deficientemente Efectivo \\
Entre 3.1 y 4.0 Puntos & Regular & Medianamente Efectivo \\
Entre 4.1 y 5.0 Puntos & Satisfactorio & Efectivo \\
\hline \hline
\end{tabular}

Fuente: construcción propia.

La Tabla 2 muestra los resultados detallados por empresa en la evaluación de cada uno de los componentes de control interno, que permiten determinar el nivel de efectividad de control interno en cada una de las mipymes objeto de estudio.

Tabla 2. Resultados de la evaluación del nivel de efectividad de control interno en cada caso

\begin{tabular}{|c|c|c|c|}
\hline Componente & $\begin{array}{l}\text { Mediana } \\
\text { Empresa }\end{array}$ & $\begin{array}{l}\text { Pequeña } \\
\text { Empresa }\end{array}$ & Micro Empresa \\
\hline Ambiente de Control & 3.7 & 3.0 & 3.3 \\
\hline Evaluación de Riesgos & 2.8 & 2.5 & 1.8 \\
\hline Actividades de Control & 3.2 & 3.0 & 2.2 \\
\hline Información y Comunicación & 3.6 & 3.3 & 3.0 \\
\hline Monitoreo & 3.3 & 2.6 & 2.7 \\
\hline $\begin{array}{c}\text { Calificación (Media } \\
\text { General) }\end{array}$ & 3.3 & 2.9 & 2.6 \\
\hline $\begin{array}{l}\text { Nivel de Efectividad del } \\
\text { Control Interno }\end{array}$ & $\begin{array}{l}\text { Medianamente } \\
\text { Efectivo }\end{array}$ & $\begin{array}{l}\text { Deficientemente } \\
\text { Efectivo }\end{array}$ & $\begin{array}{c}\text { Deficientemente } \\
\text { Efectivo }\end{array}$ \\
\hline
\end{tabular}

Fuente: construcción propia. 
En esta investigación se entendió la efectividad como el resultado caracterizado por el logro de unos objetivos acordes con lo planeado, dentro de un marco flexible y en un entorno de incertidumbre (Palma, 1993). La efectividad del control interno se mide a lo largo del tiempo. La determinación de cuándo un sistema de control interno es efectivo es un juicio que resulta de evaluar si los cinco componentes se dan y si funcionan correctamente. (COSO, 1997)

Con respecto a la efectividad del control interno en la mediana empresa del estudio, se observa que los componentes que se encuentran con un mediano control interno son el ambiente de control, las actividades de control, la información y comunicación, y el monitoreo. En el nivel más bajo de control se encuentra la valoración de riesgos, con una efectividad deficiente. En cuanto a la valoración de riesgos, la empresa se encuentra en un proceso de adecuación a la nueva estructura organizativa y son grandes los esfuerzos que se han realizado para valorar e identificar los riesgos; sin embargo, aún hace falta su constitución formal y enfocar los riesgos globales tanto internos como externos, como también en las actividades y áreas. En la empresa se han identificado y estimado los riesgos en las áreas de producción y financiera, pero en las demás áreas hace falta toda su implementación. Los riesgos deben identificarse, cuantificarse y evaluarse.

En los demás componentes de control interno para esta empresa es característico el nivel de informalidad, es decir que no existe un código de conducta claramente definido ni un reglamento interno de trabajo que constituya para todos los niveles de la empresa un sólido fundamento moral para su conducción y operación. No se ha implementado un manual de políticas que describa los lineamientos que deben seguirse en la empresa y los medios para lograr los objetivos.

No hay manuales de funciones ni de procedimientos que expongan en detalle las descripciones del trabajo, definiciones de autoridad, funciones y responsabilidades. Un manual de procedimientos que ofrezca una guía de trabajo y se describan las etapas que deben seguirse en una tarea, en donde se especifiquen los documentos soporte y la autorización requerida.

Para el elemento monitoreo en esta empresa los encargados de cada área evalúan periódicamente la eficacia en su sistema de control interno y comunican los resultados en las reuniones que se hacen semanalmente con la gerencia, no obstante, es necesario el establecimiento de informes e indicadores escritos donde se concreten el alcance de la evaluación, los objetivos a cumplir y las áreas de atención de riegos más significativas.

En la pequeña empresa, el nivel de efectividad del control interno es deficiente para todos los componentes excepto para el componente información y comunicación, que tiene un control interno medianamente efectivo. En la empresa no se le concede al control interno el carácter estratégico que requiere, no existe documentación formal de las políticas 


\section{Contex to castañeda, L.}

y procesos ni manuales de procedimientos ni funciones que permitan la implementación de políticas de control interno y de cada una de sus normas en todos los procesos de la organización. Hay ausencia de un sistema de control interno favorable, basado en indicadores, que evidencie el proceso de mejora de la organización. No hay una adecuada segregación de tareas y responsabilidades, se evidencia que los responsables de las diferentes áreas en la empresa no hacen seguimiento y revisión periódica de cada uno de los procesos que dirigen, en donde se evalúen la calidad, el cumplimiento y los resultados obtenidos y se comparen con períodos anteriores, y no hay control físico de los activos ni se elaboran presupuestos dirigidos a cumplir metas previstas.

En la microempresa la efectividad del control interno es deficiente para los componentes: actividades de control, información y comunicación, y monitoreo. El componente evaluación de riesgos se encuentra en el rango inefectivo y el ambiente de control presenta un control interno medianamente efectivo. La empresa no cuenta con objetivos y estrategias definidos, las decisiones se toman a medida que va evolucionando la empresa, no se establecen metas anualmente ni presupuestos que permitan cuantificar la producción estimada y hacer control financiero, no hay un adecuado sistema de valoración de riesgos que permita identificar, prevenir o detectar posibles errores o fraudes.

En la organización no existe revisión sistemática en cada una de las áreas claves, no todo el personal está calificado para ejecutar sus funciones. La comunicación dentro de la organización es poco eficiente y este es un factor muy importante, porque de él depende que se tomen decisiones, se resuelvan problemas, se promuevan acciones y se determinen comportamientos. Para esta empresa es necesario implementar la formalización en cada uno de sus procesos con la documentación referente a su sistema de control interno, teniendo en cuenta la formulación de políticas, la estructura organizacional y los procedimientos de control.

El resultado obtenido en relación con la efectividad del sistema de control interno en cada una de las mipymes objeto de estudio del sector confección de la ciudad de Medellín, demuestra un sistema de control interno deficiente, debido a que en estas empresas los controles se fundamentan en la experiencia empírica de quienes las administran y dirigen, y quienes además no poseen el carácter metodológico y formal que se requiere para llegar a la evaluación permanente del cumplimiento de los objetivos organizacionales.

De acuerdo con los resultados evidenciados, se puede determinar que ninguna empresa de la muestra evaluada puede garantizar la razonable confianza en su sistema de control interno que permita proteger sus activos, y verificar la exactitud y confiabilidad de la información financiera, la consecución de los objetivos de eficiencia y eficacia en sus operaciones y el cumplimiento de normas y leyes aplicables. Un sistema de control interno inefectivo o poco confiable representa un aspecto negativo para la empresa, ya que se puede 
correr el riesgo de tener desviaciones en las operaciones y la información, tomar decisiones inadecuadas en cuanto a la gestión de la empresa y tener riesgo de pérdidas financieras o no llegar a preverlas.

\section{Conclusiones}

El control interno diseñado, implementado y monitoreado de manera ordenada, eficiente y uniforme de acuerdo con las normas y procedimientos establecidos, contribuye al cumplimiento de los objetivos estratégicos y operativos en las organizaciones. Es necesario que la administración comprenda que el control interno es un proceso que genera valor a la empresa, da certeza en cuanto al aseguramiento de los procesos y la calidad en ellos y contribuye al cumplimiento de los objetivos de crecimiento, sostenibilidad y generación de valor. Las políticas de control interno para las empresas deben ser parte de las buenas prácticas corporativas y no simplemente mecanismos de control legal.

Un sistema de control interno inefectivo o poco confiable representa un aspecto negativo para la empresa, frente a eventuales desviaciones en las operaciones y en la generación de información, lo que conduce a tomar decisiones inadecuadas en cuanto a la gestión de la empresa, con pérdidas financieras que afecten su continuidad.

En este trabajo se identificó cómo las mipymes pueden pasar de un estado de informalidad y flexibilidad en los procesos de planificación y control, hasta una fase de mayor formalidad, con el establecimiento de procesos y procedimientos, a medida que la organización va creciendo y cumpliendo sus objetivos misionales. Las mipymes no cuentan con un código de conducta formal que fomente las actitudes y comportamientos adecuados de los empleados y directivos, sin embargo, la administración de las empresas demuestra su compromiso hacia el comportamiento integral y ético, comunicado a todos los empleados, lo que contribuye significativamente a propiciar un ambiente de control efectivo.

La estructura organizacional y la asignación de autoridad y responsabilidad de las empresas son adecuadas al tamaño y número de empleados, y las decisiones están dadas por los directivos de la empresa. En ninguno de los casos estudiados se cuenta con políticas y procedimientos para contratar, entrenar, promocionar y compensar a los empleados, lo que demuestra debilidades en la administración del recurso humano. El control se ejerce por costumbre y experiencia, por tanto hace falta educación y profundización sobre los componentes de un sistema de control.

En estas empresas no se han definido apropiadamente los objetivos de control que deben ser específicos, adecuados, completos, razonables e integrados a sus objetivos globales, lo que permite limitar los riesgos. En estas organizaciones no se han identificado y analizado los riesgos relevantes. El proceso de identificación y análisis de riesgos no existe en los casos 


\section{Contekto}

estudiados, de manera incipiente existe una valoración de riesgos con base en la experiencia de los administradores. El control se ejerce únicamente para el efectivo y, en algunos casos, como en la mediana empresa, se protegen los activos mediante pólizas de seguros.

La estructura organizacional es adecuada y conveniente. Las tareas y responsabilidades se relacionan con la revisión, autorización y registro de las transacciones, y están asignadas a personas diferentes. Estas entidades, por su tamaño, no deben manejar informalmente sus actividades de control. Es preciso que los empleados conozcan sus funciones y competencias, razón por la cual debe existir un manual de procedimientos y funciones formalizado. En la mediana y micro empresas, los responsables de las diferentes áreas no hacen seguimiento y revisión, como tampoco comprobación de transacciones en cuanto a exactitud, totalidad, autorización pertinente y adecuada segregación de funciones.

En cuanto a la comunicación interna, por su fácil abordaje, su estructura de pocos niveles y tamaño, en estas empresas se puede establecer comunicación directa con los administradores y propietarios. Se evidencia la existencia de líneas de comunicación abierta y la voluntad de los administradores de escuchar a sus empleados, situación que favorece la comunicación externa e interna. La comunicación entre las diferentes áreas de las empresas es buena. Las actividades se comunican de manera oportuna y con fluidez, lo que permite aumentar la coordinación entre las diferentes áreas y generar una comunicación confiable. La información es oportuna y relevante, se procesa y transmite en todos los niveles de la organización. El personal conoce la relación que hay entre sus actividades y funciones con relación al trabajo de otros y entregan los informes de manera eficiente. Se establecen reuniones grupales, especialmente en el área de producción, lo que permite que todos conozcan el proceso y se involucren con él.

De acuerdo con la investigación, el Modelo COSO puede utilizarse como una herramienta de apoyo a la gestión organizacional, independiente del tamaño de la empresa, puede ser grande, mediana, pequeña o micro. Un adecuado sistema de control interno, eficaz e integral contribuye estratégica y operativamente al logro de los objetivos misionales. Es importante señalar que, tal como lo menciona el Informe COSO en su definición, el control interno puede darles a quienes administran la organización una seguridad razonable pero no absoluta del logro de los objetivos planteados. Es un instrumento de gran valor, aunque su utilización no garantiza que se conseguirán las metas propuestas. Las decisiones en las empresas las toman los directivos, y no dependen de la estructura de control interno. Se debe tener en cuenta, además, que el control interno no remplaza la administración, constituye sólo una parte del conjunto de herramientas que permiten tomar mejores y más acertadas decisiones.

Se concluye que, para las organizaciones pequeñas, un adecuado sistema de control interno que comprenda el plan de la organización y las medidas 
adoptadas para salvaguardar los activos y verificar la exactitud de los datos contables, permite la consecución de los objetivos de eficiencia y eficacia en sus operaciones y el cumplimiento de normas y leyes, promueve la eficiencia de las operaciones, estimula la observación de políticas prescritas y logra el cumplimiento de los objetivos propuestos de la organización. El control interno es una herramienta de aplicación en todas las empresas y procesos porque ofrece la metodología necesaria para cumplir y evaluar el logro de los objetivos.

Un control interno adecuado permite a la gerencia asegurarse de que todo se encuentra en orden. Si los controles son efectivos y si se aplican ordenadamente se garantiza que las funciones se cumplan de acuerdo con las expectativas planeadas, e igualmente se identificarían las fallas que pudieran existir con el fin de tomar medidas y corregirlas. La administración debe asumir este rol del control interno como una función inherente al proceso de dirección. El control interno debe incorporarse a todas las actividades de gestión (planificación, ejecución y supervisión) para que influya en el cumplimiento de objetivos de la empresa.

Con respecto a la responsabilidad de la efectividad de un sistema de control interno adecuado a las necesidades de la organización, no sólo basta con diseñarlo, implementarlo y comunicarlo, se requiere el compromiso de todos los empleados y participantes, para que se cumpla con la premisa "contribuir con los objetivos de la organización".

\section{Referencias bibliográficas}

Andriani, C., Biasca, R. \& Rodríguez, M. (2004).Un nuevo sistema de gestión para lograr Pymes de clase mundial. 2. ${ }^{a}$ ed. Bogotá: Grupo Editorial Norma.

Beltrán, A., Torres, B., Camargo, R. \& Bello, P. C. (2004). Pymes: Un reto a la competitividad. Bogotá: Universidad Externado de Colombia.

Bernal, C. (2006). Metodología de la investigación. México: Prentice Hall.

Cámara de Comercio de Medellín para Antioquia. (2009). Avances de la estrategia Clúster en Medellín y Antioquia. Documentos Comunidad Clúster, N. ${ }^{\circ} 5$. Medellín, CCMA.

Canetti, M. (2012). Del control interno a la administración de riesgos. Buenos Aires, Argentina: Editorial La Ley.

Committee of Sponsoring Organizations of the Treadway Commission - COSO -. (1997). Internal Control - Integrated Framework. NewYork.

Committee of Sponsoring Organizations of the Treadway Commission - COSO -. (2008). Control Interno - Informe COSO. Traducción de S. Mantilla. 4. ${ }^{a}$ ed. Bogotá: Editorial Ecoe Ediciones. 


\section{Contex to castañeda, L.}

Correa, J. \& Jaramillo, F. (2007). Una aproximación metodológica y prospectiva a la gestión financiera en las pequeñas empresas. Revista Contaduría Universidad de Antioquia, 50, 98-118.

Davis, H. \& Militello, F. (1994). The empowered organization: Redefining roles y practices of finance. New Jersey: Financial Executives Research Foundation.

Dorta, V. (2005). Teorías organizativas y los sistemas de control interno. Revista Internacional Legis de Contabilidad \& Auditoría, 22, 9-58.

Ferraro, C. (2011). PYMES: Políticas de Fomento en América Latina y el Caribe. Políticas e instituciones de apoyo a las pymes en Colombia. Naciones Unidas.

Fundación Corona. (2005). Retos y desafíos del sector de la microempresa en Colombia: tres aportes conceptuales. Documento de trabajo N. ${ }^{\circ} 13$.

Hourcle, R. (2000). Auditoría. Temas seleccionados. Buenos Aires, Argentina: Ediciones Macchi.

Palma, A. (1993). Dirección de organizaciones. Santiago de Chile: Ediciones Dolmen.

Puyana, S. \& Matallana, V. (2002). Las Mipymes colombianas y el mercado internacional. Revista Civilizar, 2, 67-73.

Salkind, N. (1998). Métodos de investigación. México: Prentice Hall.

Vargas, H. (2003). Consideraciones prácticas para la gestión del capital de trabajo en las Mipymes innovadoras. Revista Escuela de Administración de Negocios, 47, 98-109.

Villegas, D. \& Toro, I. (2010). Las Pymes: Una mirada a partir de la experiencia académica del MBA. Revista MBA EAFIT, 01, 86-101.

Viloria, N. (2005). Factores que inciden en el sistema de control interno de una organización. Revista Actualidad Contable. Faces, 8 (11), 87-92.

\section{Para citar este artículo:}

Castañeda, L. (2014). Los sistemas de control interno en las Mipymes y su impacto en la efectividad empresarial. En-Contexto, 2, 129-146.

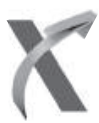

